\title{
A continuous model for quasinilpotent operators
}

\author{
Eva A. Gallardo-Gutiérrez ${ }^{1}$ • Jonathan R. Partington² ${ }^{2}$ \\ Daniel J. Rodríguez ${ }^{3}$
}

Received: 29 January 2015 / Accepted: 13 April 2016 / Published online: 11 May 2016

(C) The Author(s) 2016. This article is published with open access at Springerlink.com

\begin{abstract}
A classical result due to Foias and Pearcy establishes a discrete model for every quasinilpotent operator acting on a separable, infinite-dimensional complex Hilbert space $\mathcal{H}$. More precisely, given a quasinilpotent operator $T$ on $\mathcal{H}$, there exists a compact quasinilpotent operator $K$ in $\mathcal{H}$ such that $T$ is similar to a part of $K \oplus K \oplus \cdots \oplus K \oplus \cdots$ acting on the direct sum of countably many copies of $\mathcal{H}$. We show that a continuous model for any quasinilpotent operator can be provided. The consequences of such a model will be discussed in the context of $C_{0}$-semigroups of quasinilpotent operators.
\end{abstract}

Keywords Quasinilpotent operators $\cdot C_{0}$-semigroup · Operator model $\cdot$ Entire function

Mathematics Subject Classification Primary 47B38

\section{Introduction}

Whether every quasinilpotent operator on a separable, infinite-dimensional complex Hilbert space has a non-trivial closed invariant subspace is, by now, a long-standing open question which have called the attention of many operator theorists in last half-century. By Gel'fand's spectral radius formula, a quasinilpotent operator can be defined either as one having only 0 in its spectrum or as an operator such that the sequence of $n$th roots of norms of its $n$th powers converges to zero. Most of the known techniques for producing invariant subspaces

The authors are partially supported by Plan Nacional I+D Grant No. MTM2013-42105-P.

Jonathan R. Partington

J.R.Partington@leeds.ac.uk

1 Departamento de Análisis Matemático, Facultad de Ciencias Matemáticas, Universidad Complutense de Madrid e ICMAT, Plaza de Ciencias 3, 28040 Madrid, Spain

2 School of Mathematics, University of Leeds, Leeds LS2 9JT, United Kingdom

3 Departamento de Matemáticas, Facultad de Ciencias, Universidad de Zaragoza, Plaza San Francisco s/n, 50009 Zaragoza, Spain 
require the operator to have more than one point in its spectrum (and satisfy other conditions which involves the growth of the resolvent). Even in the case of compact operators, the proof of the existence of invariant subspaces reduces to the quasinilpotent case (non-quasinilpotent compact operators have, obviously, eigenspaces).

It has now been more than four decades since Lomonosov [6] proved a positive result about the existence of non-trivial closed invariant subspaces for operators commuting with a non-zero compact operator. That there exist quasinilpotent operators not commuting with any non-zero compact operator in a Hilbert space was already shown in the classical paper by Foias and Pearcy [4] (see also [5] for an operator not satisfying Lomonosov hypotheses).

Indeed, Foias and Pearcy's result follows from the existence of a discrete model established in [4] for quasinilpotent operators. More precisely, in [4, Theorem 1], they prove that given any bounded linear quasinilpotent (but not nilpotent) operator $T$ on a separable, infinite-dimensional complex Hilbert space $\mathcal{H}$, there exists a compact quasinilpotent weighted backward shift $K$ in $\mathcal{H}$ such that $T$ is similar to a part of $K \oplus K \oplus \cdots \oplus K \oplus \cdots$ acting on the direct sum of countably many copies of $\mathcal{H}$. That is, $T$ is similar to the restriction of $K \oplus K \oplus \cdots \oplus K \oplus \cdots$ to one of its closed invariant subspaces.

The aim of this work is taking further the study of models associated to quasinilpotent operators in order to have a better understanding of such operators. In particular, in Theorem 2.1, Sect. 2, we will prove the existence of a continuous model for a given quasinilpotent operator $T$ in the sense that there exists a $\mathcal{C}^{\infty}$ increasing positive function $w$ in $\mathbb{R}_{+}$such that $T$ is similar to the restriction of the backward shift operator $S^{*}$ to one of its closed invariant subspaces $\mathcal{M}$ in $L^{2}\left(\mathbb{R}_{+}, w(t) d t\right)$. In addition, by means of the Paley-Wiener Theorem, we will establish the counterpart of this result in a Hilbert space of entire functions.

Finally, in Sect. 3, some consequences of the existence of this continuous model will be shown in the context of $C_{0}$-semigroups of quasinilpotent operators.

\section{The model}

In this section, we show a continuous model for any quasinilpotent operator acting on a separable, infinite-dimensional complex Hilbert space $\mathcal{H}$. Before going further, we recall a few basic facts.

Given a positive decreasing function $\tilde{w}$ in $\mathbb{R}_{+}$, the space $L^{2}\left(\mathbb{R}_{+}, \tilde{w}(t) d t\right)$ consists of measurable functions in $\mathbb{R}_{+}$such that the norm

$$
\|f\|_{\tilde{w}}=\left(\int_{\mathbb{R}_{+}}|f(t)|^{2} \tilde{w}(t) d t\right)^{1 / 2}
$$

is finite. The shift operator $S$ on $L^{2}\left(\mathbb{R}_{+}, \tilde{w}(t) d t\right)$

$$
S f(t)= \begin{cases}0 & \text { if } 0 \leq t \leq 1 \\ f(t-1) & \text { if } t>1\end{cases}
$$

is well defined and bounded. In addition, the space $L^{2}\left(\mathbb{R}_{+}, \tilde{w}(t) d t\right)$ can naturally be identified as the dual space of $L^{2}\left(\mathbb{R}_{+},(\tilde{w}(t))^{-1} d t\right)$ by means of the bilinear dual pairing

$$
\langle f, g\rangle=\int_{0}^{\infty} f(t) g(t) d t, \quad\left(f \in L^{2}\left(\mathbb{R}_{+}, \tilde{w}(t) d t\right), g \in L^{2}\left(\mathbb{R}_{+},(\tilde{w}(t))^{-1} d t\right)\right),
$$


giving an isometric isomorphism. The backward shift on $L^{2}\left(\mathbb{R}_{+},(\tilde{w}(t))^{-1} d t\right)$ is therefore the adjoint of the shift on $L^{2}\left(\mathbb{R}_{+}, \tilde{w}(t) d t\right)$ and, thus

$$
\left(S^{*} f\right)(t)=f(t+1), \quad(t>0),
$$

for $f \in L^{2}\left(\mathbb{R}_{+},(\tilde{w}(t))^{-1} d t\right)$. Moreover, it is clear that the invariant subspaces of the backward shift on $L^{2}\left(\mathbb{R}_{+},(\tilde{w}(t))^{-1} d t\right)$ can be identified as the annihilators of the invariant subspaces of the forward shift on $L^{2}\left(\mathbb{R}_{+}, \tilde{w}(t) d t\right)$. With this at hand, we are in a position to state the following

Theorem 2.1 Let $T$ be a quasinilpotent (but not nilpotent) operator defined on a separable, infinite-dimensional complex Hilbert space $\mathcal{H}$. Then there exists a positive increasing function $w \in \mathcal{C}^{\infty}\left(\mathbb{R}_{+}\right)$with $w(t)^{1 / t} \rightarrow \infty$ as $t \rightarrow \infty$ such that $T$ is similar to a part of the backward shift operator $S^{*}$ in $L^{2}\left(\mathbb{R}_{+}, w(t) d t\right)$.

Proof Firstly, let us show the existence of a positive increasing step function $\chi$ such that $T$ is similar to a part of the backward shift operator $S^{*}$ acting on $L^{2}\left(\mathbb{R}_{+}, \chi(t) d t\right)$. Proceeding as in Foias-Pearcy's Theorem, we start considering the sequences

$$
\begin{array}{ll}
\alpha_{n}=\left\|T^{n}\right\|^{1 / 2}, & \left(n \in \mathbb{Z}_{+}\right), \\
\kappa_{n}^{2}=\left(\alpha_{2^{p_{n}}}^{1 / 2^{p_{n}}}\right) \alpha_{1}, & \left(n=2^{p_{n}}+q_{n}, 0 \leq q_{n}<2^{p_{n}}\right) .
\end{array}
$$

Observe that the non-negative integers $p_{n}$ and $q_{n}$ are uniquely determined for all $n \geq 1$. Moreover, the sequence $\left\{p_{n}\right\}_{n \geq 1}$ is non-decreasing and $p_{n} \rightarrow \infty$ as $n \rightarrow \infty$.

Let us fix $\kappa_{0}=1$. Since $\alpha_{2 n} \leq \alpha_{n}^{2}$ for all $n \in \mathbb{Z}_{+}$, it follows that

$$
\alpha_{2^{n+1}}^{1 / 2^{n+1}} \leq \alpha_{2^{n}}^{1 / 2^{n}}, \quad(n \geq 1) .
$$

On the other hand, the sequence $\left\{\kappa_{n}\right\}_{n \in \mathbb{Z}_{+}}$decreases to zero, since $T$ is quasinilpotent and $\left\{p_{n}\right\}_{n \geq 1}$ is a non-decreasing sequence. Therefore, if $\left\{e_{n}\right\}_{n \in \mathbb{Z}_{+}}$is an orthonormal basis of $\mathcal{H}$, then the weighted backward shift operator $K$ defined by

$$
K e_{0}=0, \quad K e_{n+1}=\kappa_{n} e_{n}, \quad\left(n \in \mathbb{Z}_{+}\right),
$$

is clearly compact and quasinilpotent. Hence, the operator

$$
\tilde{K}=K \oplus K \oplus \cdots \oplus K \oplus \cdots
$$

defined on $\mathcal{H}_{\infty}$, the direct sum of countably many copies of $\mathcal{H}$ indexed by the positive integers, is unitarily equivalent to the operator $J$ given by

$$
J\left(h_{0}, h_{1}, h_{2} \ldots\right)=\left(\kappa_{0} h_{1}, \kappa_{1} h_{2}, \ldots\right), \quad\left(h_{n} \in \mathcal{H}_{n}, n \in \mathbb{Z}_{+}\right) .
$$

Now, let us consider the following decomposition

$$
L^{2}\left(\mathbb{R}_{+}\right)=L^{2}(0,1) \oplus L^{2}(1,2) \oplus \cdots \oplus L^{2}(n, n+1) \oplus \cdots .
$$

Let denote by $U_{n}$ the unitary operator from $L^{2}(n-1, n)$ onto $\mathcal{H}_{n}$ (the $n$th copy of $\mathcal{H}$ in $\mathcal{H}_{\infty}$ ) for all $n \in \mathbb{Z}_{+}$, and define

$$
U=U_{1} \oplus \cdots \oplus U_{n} \oplus \cdots
$$

acting on $L^{2}\left(\mathbb{R}_{+}\right)$. By means of $U$, it can be proved that $\tilde{K}$ is unitarily equivalent to the operator $\tilde{S}$ on $L^{2}\left(\mathbb{R}_{+}\right)$defined by

$$
\tilde{S} f(t)=\lambda(t)\left(S^{*} f\right)(t)=\lambda(t) f(t+1), \quad\left(t \in \mathbb{R}_{+}\right),
$$


where $\lambda$ is the positive non-decreasing step function given by

$$
\lambda(t)=\kappa_{\lfloor t\rfloor}, \quad\left(t \in \mathbb{R}_{+}\right) .
$$

Observe that $\lambda(t)=1$ for $0<t<1$, since $\kappa_{0}=1$ was previously fixed. Now, let $\left\{w_{n}\right\}_{n \geq 0}$ be the sequence

$$
w_{0}=1, \quad w_{n+1}=\left(\kappa_{0}^{2} \kappa_{1}^{2} \cdots \kappa_{n}^{2}\right)^{-1}, \quad\left(n \in \mathbb{Z}_{+}\right) .
$$

Note that $w(n)^{1 / n} \rightarrow \infty$ as $n \rightarrow \infty$. Now, we define the positive step function

$$
\chi(t)=w_{\lfloor t\rfloor}, \quad\left(t \in \mathbb{R}_{+}\right) .
$$

Observe that $\chi$ is a non-decreasing function. Moreover, the operator

$$
V f(t)=\frac{1}{\sqrt{\chi(t)}} f(t), \quad\left(t \in \mathbb{R}_{+}\right),
$$

induces a unitary equivalence between $L^{2}\left(\mathbb{R}_{+}\right)$and the weighted space $L^{2}\left(\mathbb{R}_{+}, \chi(t) d t\right)$ such that for every $f \in L^{2}\left(\mathbb{R}_{+}, \chi(t) d t\right)$ we have

$$
V \tilde{S} V^{*} f(t)=\lambda(t) \sqrt{\frac{\chi(t+1)}{\chi(t)}} f(t+1)=f(t+1), \quad\left(t \in \mathbb{R}_{+}\right) .
$$

Therefore, the operator $\tilde{S}$ in $L^{2}\left(\mathbb{R}_{+}\right)$is unitarily equivalent to $S^{*}$ in $L^{2}\left(\mathbb{R}_{+}, \chi(t) d t\right)$. Combining this with the fact $T$ is similar to a part of $\tilde{K}$ acting on $\mathcal{H}_{\infty}$, we conclude that $T$ is similar to a part of the backward shift operator $S^{*}$ acting on $L^{2}\left(\mathbb{R}_{+}, \chi(t) d t\right)$.

It remains to prove that $\chi$ may be regularized into a $\mathcal{C}^{\infty}$-function in $\mathbb{R}_{+}$. Observe that

$$
\chi(t)=\sum_{n \geq 0} w_{n} \chi_{[n, n+1)}(t), \quad\left(t \in \mathbb{R}_{+}\right),
$$

where $\chi_{[n, n+1)}$ is the characteristic function of the interval $[n, n+1), n \geq 0$. For a fixed $\varepsilon>0$, let $\alpha$ be a non-decreasing $\mathcal{C}^{\infty}$-function in the interval $[0, \varepsilon]$ such that

$$
\alpha(0)=0, \quad \alpha(\varepsilon)=w_{1}-w_{0}=w_{1}-1 .
$$

Roughly speaking, $\alpha$ joins the values 0 and $w_{1}-1$ by a $\mathcal{C}^{\infty}$-function. Now, let $w$ be the non-decreasing function in $\mathcal{C}^{\infty}\left(\mathbb{R}_{+}\right)$defined by

$$
w(t)=\sum_{n=0}^{\infty} W_{n}(t) \chi_{[n, n+1)}(t), \quad\left(t \in \mathbb{R}_{+}\right),
$$

where, for each $n \in \mathbb{Z}_{+}$, the function $W_{n}$ is given by

$$
W_{n}(t)= \begin{cases}w_{n} & \text { if } n \leq t<(n+1)-\varepsilon, \\ w_{n}+\frac{w_{n+1}-w_{n}}{w_{1}-w_{0}} \alpha(t-(n+1-\varepsilon)) & \text { if }(n+1)-\varepsilon \leq t<(n+1) .\end{cases}
$$

Note that $w(t)^{1 / t} \rightarrow \infty$ as $t \rightarrow \infty$. 
Now, observe that $f \in L^{2}\left(\mathbb{R}_{+}, \chi(t) d t\right)$, for all $f \in L^{2}\left(\mathbb{R}_{+}, w(t) d t\right)$. On the other hand, if $f \in L^{2}\left(\mathbb{R}_{+}, \chi(t) d t\right)$, then $S^{*} f \in L^{2}\left(\mathbb{R}_{+}, \chi(t) d t\right)$. Moreover, since

$$
\begin{aligned}
\left\|S^{*} f\right\|_{w}^{2} & \leq\left\|S^{*} f\right\|_{\chi}^{2}+\sum_{n=0}^{\infty} \int_{[n+1-\varepsilon, n+1)}|f(t+1)|^{2} W_{n}(t) d t \\
& \leq\left\|S^{*} f\right\|_{\chi}^{2}+\int_{[1-\varepsilon, 1)}|f(t+1)|^{2} W_{0}(t) d t+\sum_{n=1}^{\infty} \int_{[n+2-\varepsilon, n+2)}|f(t)|^{2} w_{n+1} d t \\
& \leq\left\|S^{*} f\right\|_{\chi}^{2}+\int_{[1-\varepsilon, 1)}|f(t+1)|^{2} W_{0}(t) d t+\|f\|_{\chi}^{2},
\end{aligned}
$$

it follows that $S^{*} f \in L^{2}\left(\mathbb{R}_{+}, w(t) d t\right)$, for all $f \in L^{2}\left(\mathbb{R}_{+}, \chi(t) d t\right)$. From here, the statement of Theorem 2.1 follows.

\subsection{A Hilbert space of entire functions associated to the continuous model for quasinilpotent operators}

In this subsection, we show that it is possible to associate a Hilbert space of entire functions to the continuous model of a quasinilpotent operator by means of a theorem of Paley-Wiener.

Let $\mathbb{C}_{+}$denote the open right-half complex plane. Recall that the Hardy space $\mathcal{H}^{2}\left(\mathbb{C}_{+}\right)$ consists of the functions $F$ analytic on $\mathbb{C}_{+}$with finite norm

$$
\|F\|_{\mathcal{H}^{2}\left(\mathbb{C}_{+}\right)}=\left\{\frac{1}{2 \pi} \sup _{0<x<\infty} \int_{-\infty}^{\infty}|F(x+i y)|^{2} d y\right\}^{1 / 2} .
$$

A classical theorem of Paley and Wiener (see [8], for instance) states that $\mathcal{H}^{2}\left(\mathbb{C}_{+}\right)$is isometrically isomorphic under the Laplace transform to $L^{2}\left(\mathbb{R}_{+}\right)$, the space of functions square-integrable over the positive real line. In fact, to each function $F \in \mathcal{H}^{2}\left(\mathbb{C}_{+}\right)$there corresponds a function $f \in L^{2}\left(\mathbb{R}_{+}\right)$such that

$$
F(s)=(\mathcal{L} f)(s):=\int_{0}^{\infty} f(t) e^{-s t} d t, \quad\left(s \in \mathbb{C}_{+}\right),
$$

and

$$
\|F\|_{\mathcal{H}^{2}\left(\mathbb{C}_{+}\right)}^{2}=\int_{0}^{\infty}|f(t)|^{2} d t .
$$

Given a quasinilpotent (but not nilpotent) operator $T$ defined on a separable, infinitedimensional complex Hilbert space $\mathcal{H}$, let $\left(S^{*} \mid \mathcal{M}, \chi\right)$ denote the model for the operator $T$ provided by Theorem 2.1, where $\chi$ is the function defined by Eq. (4). Now, for every function $f \in L^{2}\left(\mathbb{R}_{+}, \chi(t) d t\right)$, we consider the following decomposition

$$
f=f_{0}+f_{1}+f_{2}+\cdots,
$$

where $f_{n} \in L^{2}\left([n, n+1), w_{n} d t\right)$ for all $n \in \mathbb{Z}_{+}$. The Laplace transform of $f$ has the form

$$
\mathcal{L} f(s)=F(s)=\int_{0}^{\infty} f(t) e^{-s t} d t=\sum_{n=0}^{\infty} e^{-n s} F_{n}(s), \quad\left(s \in \mathbb{C}_{+}\right),
$$

where, for each $n \in \mathbb{Z}_{+}$, the function $F_{n}$ is given by

$$
F_{n}(s)=\int_{0}^{1} f_{n}(n+t) e^{-s t} d t, \quad\left(s \in \mathbb{C}_{+}\right) .
$$


In fact, the series in (6) converges for all $s \in \mathbb{C}$. This is just a consequence of the CauchySchwarz inequality

$$
|F(s)|=\left|\sum_{n \in \mathbb{Z}_{+}} e^{-n s} F_{n}(s)\right| \leq\left(\sum_{n \in \mathbb{Z}_{+}} \frac{e^{-2 n \Re \mathfrak{e} s}}{w_{n}}\right)^{1 / 2}\|f\|_{\chi}, \quad(s \in \mathbb{C}),
$$

which, combined with the fact that the weighted backward shift $K$ defined in (1) is quasinilpotent, imply the convergence for all $s \in \mathbb{C}$ of the series

$$
\sum_{n=1}^{\infty} \frac{e^{-2 n \mathfrak{R e} s}}{w_{n}}=\sum_{n=1}^{\infty}\left(\kappa_{0}^{2} \kappa_{1}^{2} \cdots \kappa_{n-1}^{2}\right) e^{-2 n \Re \mathfrak{e} s} .
$$

An application of Morera's Theorem shows that $F$ is an entire function.

With this at hand, we rewrite Theorem 2.1 in the context of analytic functions as follows.

Theorem 2.2 Let $T$ be a quasinilpotent (but not nilpotent) operator on a separable, infinitedimensional complex Hilbert space $\mathcal{H}$. Then there exists a subspace $\mathcal{Y}$ of the Hardy space $\mathcal{H}^{2}\left(\mathbb{C}_{+}\right)$consisting of entire functions such that $T$ is similar to the restriction of the adjoint of the multiplication operator by the symbol $\phi(s)=e^{-s}, s \in \mathbb{C}$, to one of its closed invariant subspaces $\mathcal{M}$ in $\mathcal{Y}$.

Remark 2.3 Observe that $\mathcal{H}^{2}\left(\mathbb{C}_{+}\right)$is a functional Hilbert space, and therefore the reproducing kernels play an important role in order to determine non-trivial closed invariant subspaces of the adjoint of the multiplication operator by the symbol $\phi(s)=e^{-s}, s \in \mathbb{C}$.

Remark 2.4 By means of the function $\chi$, the norm of every function in $L^{2}\left(\mathbb{R}_{+}, \chi(t) d t\right)$ may be expressed in terms of the Laplace transform of it as well of its shifts, that is

$$
\begin{aligned}
\|f\|_{\chi}^{2} & =\int_{0}^{\infty}|f(t)|^{2} \chi(t) d t=\sum_{k=0}^{\infty}\left(\int_{k}^{k+1}|f(t)|^{2} w_{k} d t\right) \\
& =\int_{0}^{\infty}|f(t)|^{2} d t+\sum_{k=1}^{\infty}\left(\int_{k}^{\infty}|f(t)|^{2}\left(w_{k}-w_{k-1}\right) d t\right) \\
& =\|f\|_{L^{2}\left(\mathbb{R}_{+}\right)}^{2}+\sum_{k=1}^{\infty}\left(w_{k}-w_{k-1}\right)\left\|\left(S^{*}\right)^{k} f\right\|_{L^{2}\left(\mathbb{R}_{+}\right)}^{2} \\
& =\|\mathcal{L} f\|_{\mathcal{H}^{2}\left(\mathbb{C}_{+}\right)}^{2}+\sum_{k=1}^{\infty}\left(w_{k}-w_{k-1}\right)\left\|\mathcal{L}\left(\left(S^{*}\right)^{k} f\right)\right\|_{\mathcal{H}^{2}\left(\mathbb{C}_{+}\right)}^{2}
\end{aligned}
$$

where the last equality follows as an application of the Paley-Wiener Theorem.

We end up the section with two examples, where it is possible to determine explicitly the subspace $\mathcal{Y}$ stated in Theorem 2.2.

Example 2.5 The space $L^{2}\left(\mathbb{R}_{+}, e^{t^{2}} d t\right)$ is isometrically isomorphic under the Laplace transform to a proper subspace $\mathcal{Y}$ of entire functions of $\mathcal{H}^{2}\left(\mathbb{C}_{+}\right)$with the norm

$$
\|\mathcal{L} f\|_{\mathcal{Y}}^{2}=\sum_{n=0}^{\infty} \frac{\left\|(\mathcal{L} f)^{(n)}\right\|_{\mathcal{H}^{2}\left(\mathbb{C}_{+}\right)}^{2}}{n !}
$$


where, for each $n \in \mathbb{Z}_{+}$, the $n$th derivative of the Laplace transform of $f$ is given by

$$
(\mathcal{L} f)^{(n)}(s)=\int_{0}^{\infty}(-1)^{n} t^{n} f(t) e^{-s t} d t, \quad\left(s \in \mathbb{C}_{+}\right) .
$$

Let $f$ be a function in $L^{2}\left(\mathbb{R}_{+}, e^{t^{2}} d t\right)$. Since $(-1)^{n} t^{n} f(t) \in L^{2}\left(\mathbb{R}_{+}\right)$for all $n \in \mathbb{Z}_{+}$, it follows from the Paley-Wiener Theorem that both $\mathcal{L} f$ and all its derivatives belong to $\mathcal{H}^{2}\left(\mathbb{C}_{+}\right)$with

$$
\left\|(\mathcal{L} f)^{(n)}\right\|_{\mathcal{H}^{2}\left(\mathbb{C}_{+}\right)}^{2}=\int_{0}^{\infty} t^{2 n}|f(t)|^{2} d t, \quad\left(n \in \mathbb{Z}_{+}\right) .
$$

Therefore

$$
\|f\|_{L^{2}\left(\mathbb{R}_{+}, e^{t^{2}} d t\right)}^{2}=\int_{0}^{\infty}|f(t)|^{2} e^{t^{2}} d t=\int_{0}^{\infty}|f(t)|^{2}\left(\sum_{n=0}^{\infty} \frac{t^{2 n}}{n !}\right) d t=\sum_{n=0}^{\infty} \frac{\left\|(\mathcal{L} f)^{(n}\right\|_{\mathcal{H}^{2}\left(\mathbb{C}_{+}\right)}^{2}}{n !} .
$$

Finally, since $f$ belongs to the space $\in L^{2}\left(\mathbb{R}_{+}, e^{t^{2}} d t\right)$, for every $s \in \mathbb{C}$ we have

$$
|\mathcal{L} f(s)|=\left|\int_{0}^{\infty} f(t) e^{-s t} d t\right| \leq\|f\|_{L^{2}\left(\mathbb{R}_{+}, e^{t^{2}} d t\right)}\left(\int_{0}^{\infty} e^{-t(t+2 \Re \mathfrak{R} s)} d t\right)^{1 / 2}<\infty .
$$

As an application of Morera's Theorem, it follows that $\mathcal{L} f$ is analytic in $\mathbb{C}$.

Our next example is a generalization of the previous one. In order to state it, just recall that the Bergman space $\mathcal{A}^{2}\left(\mathbb{C}_{+}\right)$consists of those functions $F$ which are analytic on $\mathbb{C}_{+}$with finite norm

$$
\|F\|_{\mathcal{A}^{2}\left(\mathbb{C}_{+}\right)}=\left\{\frac{1}{\pi} \int_{\mathbb{C}_{+}}|F(x+i y)|^{2} d x d y\right\}^{1 / 2} .
$$

A Paley-Wiener Theorem for $\mathcal{A}^{2}\left(\mathbb{C}_{+}\right)$states that the Laplace transform $\mathcal{L}$ is an isometric isomorphism between $L^{2}\left(\mathbb{R}_{+}, t^{-1} d t\right)$ and $\mathcal{A}^{2}\left(\mathbb{C}_{+}\right)$(see [1], for instance).

Proposition 2.6 Suppose $w(t)=\sum_{n \in \mathbb{Z}_{+}} a_{n} t^{n}$ with $a_{0} \neq 0$ and $a_{n} \geq 0$ for all $n \in \mathbb{Z}_{+}$. Then the space $L^{2}\left(\mathbb{R}_{+}, w(t) d t\right)$ is isometrically isomorphic under the Laplace transform to a proper subspace $\mathcal{Y}$ of $\mathcal{H}^{2}\left(\mathbb{C}_{+}\right)$with the norm

$$
\|\mathcal{L} f\|_{\mathcal{Y}}^{2}=\sum_{n \text { even }} a_{n}\left\|(\mathcal{L} f)^{(n / 2)}\right\|_{\mathcal{H}^{2}\left(\mathbb{C}_{+}\right)}^{2}+\sum_{n \text { odd }} a_{n}\left\|(\mathcal{L} f)^{\left(\frac{n+1}{2}\right)}\right\|_{\mathcal{A}^{2}\left(\mathbb{C}_{+}\right)}^{2}
$$

Proof Let $f$ be a function in $L^{2}\left(\mathbb{R}_{+}, w(t) d t\right)$. For those $n \in \mathbb{Z}_{+}$with $a_{n} \neq 0$ we have

$$
\int_{0}^{\infty} t^{n}|f(t)|^{2} d t<\infty
$$

In particular, $f \in L^{2}\left(\mathbb{R}_{+}\right)$. By the Paley-Wiener Theorem we know that $\mathcal{L} f$ belongs to $\mathcal{H}^{2}\left(\mathbb{C}_{+}\right)$. Observe that the norm of $f$ can be expressed as follows

$$
\|f\|_{w}^{2}=\int_{0}^{\infty}|f(t)|^{2} w(t) d t=\sum_{n=0}^{\infty} a_{n}\left(\int_{0}^{\infty} t^{n}|f(t)|^{2} d t\right) .
$$

Now, since $t^{n / 2} f(t) \in L^{2}\left(\mathbb{R}_{+}\right)$for all even integers $n \in \mathbb{Z}_{+}$with $a_{n} \neq 0$, we have

$$
\left\|\mathcal{L}\left(t^{n / 2} f(t)\right)\right\|_{\mathcal{H}^{2}\left(\mathbb{C}_{+}\right)}=\left\|t^{n / 2} f(t)\right\|_{L^{2}\left(\mathbb{R}_{+}\right)},
$$


where

$$
\mathcal{L}\left(t^{n / 2} f(t)\right)(s)=\int_{0}^{\infty} t^{n / 2} f(t) e^{-s t} d t=(-1)^{n / 2}(\mathcal{L} f)^{(n / 2)}(s), \quad\left(n \in \mathbb{Z}_{+}, \quad n \text { even }\right) .
$$

On the other hand, since $t^{(n+1) / 2} f(t) \in L^{2}\left(\mathbb{R}_{+}, t^{-1} d t\right)$ for all odd integers $n \in \mathbb{Z}_{+}$with $a_{n} \neq 0$, we have

$$
\left\|\mathcal{L}\left(t^{(n+1) / 2} f(t)\right)\right\|_{\mathcal{A}^{2}\left(\mathbb{C}_{+}\right)}=\left\|t^{(n+1) / 2} f(t)\right\|_{L^{2}\left(\mathbb{R}_{+}, t^{-1} d t\right)}, \quad\left(n \in \mathbb{Z}_{+}, n \text { odd }\right) .
$$

Therefore,

$$
\begin{aligned}
\|f\|_{w}^{2} & =\sum_{n \text { even }} a_{n}\left(\int_{0}^{\infty} t^{n}|f(t)|^{2} d t\right)+\sum_{n \text { odd }} a_{n}\left(\int_{0}^{\infty} t^{n}|f(t)|^{2} d t\right) \\
& =\sum_{n \text { even }} a_{n}\left\|t^{n / 2} f(t)\right\|_{L^{2}\left(\mathbb{R}_{+}\right)}^{2}+\sum_{n \text { odd }} a_{n}\left\|t^{(n+1) / 2} f(t)\right\|_{L^{2}\left(\mathbb{R}_{+}, t^{-1} d t\right)}^{2} \\
& =\sum_{n \text { even }} a_{n}\left\|(\mathcal{L} f)^{(n / 2)}\right\|_{\mathcal{H}^{2}\left(\mathbb{C}_{+}\right)}^{2}+\sum_{n \text { odd }} a_{n}\left\|(\mathcal{L} f)^{\left(\frac{n+1}{2}\right)}\right\|_{\mathcal{A}^{2}\left(\mathbb{C}_{+}\right)}^{2} \\
& =\|\mathcal{L} f\|_{\mathcal{Y}}^{2} .
\end{aligned}
$$

Finally, we apply the continuous model given by Theorem 2.1 to the study of invariant subspaces in the context of $C_{0}$-semigroups of quasinilpotent operators.

\section{$3 C_{0}$-semigroups of quasinilpotent operators}

In this section, we derive an application of Theorem 2.1 to $C_{0}$-semigroups of quasinilpotent operators. Before stating this result, let us recall some basic notions.

Let $\{T(t)\}_{t \geq 0}$ be a $C_{0}$-semigroup of operators defined on a Hilbert $\mathcal{H}$, i.e., a family of bounded linear operators satisfying the functional equation

$$
\left\{\begin{array}{l}
T(t+s)=T(t) T(s), \quad(t, s \geq 0), \\
T(0)=I
\end{array}\right.
$$

such that $\{T(t)\}_{t \geq 0}$ converges to the identity operator $I$ in the strong operator topology. In this context, it is well-known that there exists a closed and densely defined linear operator $A$ that determines the semigroup uniquely, called the generator of $\{T(t)\}_{t \geq 0}$, defined as

$$
A x:=\lim _{h \rightarrow 0^{+}} \frac{1}{h}(T(h) x-x),
$$

where its domain $D(A)$ consists of all those vectors $x \in \mathcal{H}$ for which this limit exists (see [2, Chapter II], for instance). In general, $A$ is an unbounded operator. However, if $1 \in \rho(A)$, then the transformation defined by

$$
V:=(A+I)(A-I)^{-1}
$$

is a bounded operator on $\mathcal{H}$ that determines the semigroup uniquely, since $A$ does. This operator $V$ is called the cogenerator of $\{T(t)\}_{t \geq 0}$ (see [7], for instance). Observe that 1 is not an eigenvalue of $V$. 
An immediate consequence from the above definition, using the spectral mapping theorem for the resolvent of the generator $A$ (see [2, Chapter IV,Proposition 1.13]), is a complete description for the spectrum of the cogenerator $V$ given by

$$
\sigma(V) \backslash\{1\}=\left\{\frac{\lambda+1}{\lambda-1}: \lambda \in \sigma(A)\right\} .
$$

If $\{T(t)\}_{t \geq 0}$ is a $C_{0}$-semigroup of quasinilpotent (but not nilpotent) operators, it holds that the spectrum of its generator $A$ is the empty set. Therefore, in this case, the spectrum of its cogenerator is given by $\sigma(V)=\{1\}$ (see [2, Chapter IV, Corollary 2.5]).

Applying Theorem 2.1 to $I-V$, which is quasinilpotent but not nilpotent (since 0 is not an eigenvalue), we obtain the following result.

Proposition 3.1 Let $V$ be the cogenerator of a $C_{0}$-semigroup $\{T(t)\}_{t \geq 0}$ of quasinilpotent operators on a separable, infinite-dimensional complex Hilbert space $\mathcal{H}$. Then there exists a positive increasing function $w \in \mathcal{C}^{\infty}\left(\mathbb{R}_{+}\right)$such that $V$ is similar to a part of the operator $I-S^{*}$ in $L^{2}\left(\mathbb{R}_{+}, w(t) d t\right)$, where $S^{*}$ denotes the backward shift operator.

Using a $H^{\infty}$ functional calculus, it is possible to recover each element of the semigroup by means of the cogenerator. To this end, we need $V$ to be a contraction, as in the following result.

Theorem 3.2 ([7], p. 142) Let $V$ be a contraction on a Hilbert space $\mathcal{H}$. In order that there exists a $C_{0}$-semigroup $\{T(t)\}_{t \geq 0}$ whose cogenerator equals $V$, it is necessary and sufficient that 1 is not an eigenvalue of $V$. If this is the case, then $V$ and $T(t)$ determine each other by the relations

$$
V=\lim _{t \rightarrow 0^{+}} \varphi_{t}(T(t)) \text { and } T(t)=e_{t}(V), \quad(t \geq 0)
$$

where

$$
\begin{aligned}
& e_{t}(z)=\exp \left(t \frac{z+1}{z-1}\right), \quad(t \geq 0, z \in \mathbb{D}), \\
& \varphi_{t}(z)=\frac{z-1+t}{z-1-t}=\frac{1-t}{1+t}-\frac{2 t}{1+t} \sum_{n=1}^{\infty} \frac{z^{n}}{(1+t)^{n}}, \quad(0<t<1, z \in \mathbb{D})
\end{aligned}
$$

Observe that, if $\{T(t)\}_{t \geq 0}$ is a quasinilpotent semigroup that is not a contraction semigroup, we can define a pre-Hilbert norm $|\|\cdot\|| \mid$ on $\mathcal{H}$ by

$$
\|x\|^{2}=\int_{0}^{\infty}\|T(t) x\|^{2} d t, \quad(x \in \mathcal{H}) .
$$

Let $\widetilde{\mathcal{H}}$ denote the completion of $\mathcal{H}$ with respect to $\|\cdot \cdot\| \|$, and note that $\{T(t)\}_{t \geq 0}$ extends to a quasinilpotent contraction semigroup $\{\widetilde{T}(t)\}_{t \geq 0}$ on $\mathcal{H}$, since clearly $\|T(t)\| \leq\|T(t)\|$ for all $t \geq 0$. The generators $A$ and $\widetilde{A}$ satisfy $D(A) \subseteq D(\widetilde{A})$ and $\widetilde{A}_{\mid D(A)}=A$.

The cogenerator $\widetilde{V}$ is now a contraction with spectrum equal to $\{1\}$, and thus we may apply Theorem 2.1 to $I-\widetilde{V}$ as before, to obtain the following result.

Proposition 3.3 Let $\{T(t)\}_{t \geq 0}$ be a $C_{0}$-semigroup of quasinilpotent (but not nilpotent) operators on a separable, infinite-dimensional complex Hilbert space $\mathcal{H}$, and let $\widetilde{V}$ be the cogenerator of $\{\widetilde{T}(t)\}_{t \geq 0}$, as defined above. Then there exists a positive increasing function $w \in \mathcal{C}^{\infty}\left(\mathbb{R}_{+}\right)$such that $\widetilde{V}$ is similar to the restriction of the operator $I-S^{*}$ to a closed invariant subspace $\widetilde{\mathcal{M}}$ in $L^{2}\left(\mathbb{R}_{+}, w(t) d t\right)$, where $S^{*}$ denotes the backward shift operator. Moreover, $\widetilde{T}(t)$ is similar to $e_{t}\left(I-S_{\mid \widetilde{M}}^{*}\right)$, as defined in (8). 
Finally, let us remark that Proposition 3.3 allows us just to recover the extended $C_{0^{-}}$ semigroup $\{\widetilde{T}(t)\}_{t \geq 0}$ from its cogenerator by an $H^{\infty}$ functional calculus; but in what concerns the $C_{0}$-semigroup $\{T(t)\}_{t \geq 0}$, we pose the following question:

Does there exist an increasing function $\omega \in \mathcal{C}^{\infty}\left(\mathbb{R}_{+}\right)$and an $S^{*}$-invariant subspace $\mathcal{M}$ in $L^{2}\left(\mathbb{R}_{+}, \omega(t) d t\right)$ such that $T(t)$ is similar to $e_{t}\left(I-S_{\mid \mathcal{M}}^{*}\right)$ ?

Acknowledgments The third author acknowledges Ministerio de Economía y Competitividad (Spain) for the research grant "Ayudas a la movilidad predoctoral para la realización de estancias breves en centros $I+D$ ", ref. EEBB-I-14-08134. The first and third authors are grateful to the School of Mathematics at the University of Leeds for the hospitality shown during their stay.

Open Access This article is distributed under the terms of the Creative Commons Attribution 4.0 International License (http://creativecommons.org/licenses/by/4.0/), which permits unrestricted use, distribution, and reproduction in any medium, provided you give appropriate credit to the original author(s) and the source, provide a link to the Creative Commons license, and indicate if changes were made.

\section{References}

1. Duren, P., Gallardo-Gutiérrez, E.A., Montes-Rodríguez, A.: A Paley-Wiener theorem for Bergman spaces with application to invariant subspaces. Bull. Lond. Math. Soc. 39(3), 459-466 (2007)

2. Engel, K.J., Nagel, R.: One-Parameter Semigroups for Linear Evolution Equations, Graduate Text in Mathematics, vol. 194. Springer, New York (2000)

3. Foias, C., Jung, I., Ko, E., Pearcy, C.: On quasinilpotent operators II. J. Aust. Math. Soc. 77, 349-356 (2004)

4. Foias, C., Pearcy, C.: A model for quasinilpotent operators. Mich. Math. J. 21, 399-404 (1974)

5. Hadwin, D.W., Nordgren, E.A., Radjavi, H., Rosenthal, P.: An operator not satisfying Lomonosov's hypothesis. J. Funct. Anal. 38, 410-415 (1980)

6. Lomonosov, V.: Invariant subspaces for operators commuting with compact operatos. Funct. Anal. Appl. 7, 213-214 (1973)

7. Nagy, B.Sz, Foias, C.: Harmonic Analysis of Operators on Hilbert Space. North-Holland Publishing Co., Amsterdam (1970)

8. Rudin, W.: Real and Complex Analysis, 3rd edn. McGraw-Hill, New York (1987) 